\title{
Circulating adipokines are associated with pre-eclampsia in women with type 1 diabetes
}

\author{
Clare B. Kelly ${ }^{1,2}$ • Michelle B. Hookham ${ }^{1,3}$ - Jeremy Y. Yu ${ }^{1,2} \cdot$ Samuel M. Lockhart ${ }^{1}$ • \\ Mei Du ${ }^{4}$ - Alicia J. Jenkins ${ }^{2,5}$ - Alison Nankervis ${ }^{6}$ - Kristian F. Hanssen ${ }^{7,8}$. \\ Tore Henriksen $^{8}$ • Satish K. Garg ${ }^{9}$ - Samar M. Hammad ${ }^{10}$ • James A. Scardo ${ }^{11}$. \\ Christopher E. Aston ${ }^{12}$ • Christopher C. Patterson ${ }^{13}$ • Timothy J. Lyons ${ }^{1,2}$
}

Received: 16 May 2017 / Accepted: 13 July 2017 /Published online: 5 September 2017

(C) Springer-Verlag GmbH Germany 2017

\begin{abstract}
Aims/hypothesis The incidence of pre-eclampsia, a multisystem disorder of pregnancy, is fourfold higher in type 1 diabetic than non-diabetic women; it is also increased in women with features of the metabolic syndrome and insulin resistance. In a prospective study of pregnant women with type 1 diabetes, we measured plasma levels of adipokines known to be associated with insulin resistance: leptin, fatty acid binding protein 4 (FABP4), adiponectin (total and high molecular weight [HMW]; also known as high molecular mass), retinol binding protein 4 (RBP4) and resistin and evaluated associations with the subsequent development of pre-eclampsia.

Methods From an established prospective cohort of pregnant type 1 diabetic women, we studied 23 who developed preeclampsia and 24 who remained normotensive; for reference values we included 19 healthy non-diabetic normotensive pregnant women. Plasma adipokines were measured (by ELISA) in stored samples from three study visits (Visit 1Visit 3$)$ at different gestational ages (mean \pm SD): Visit 1 ,
\end{abstract}

Timothy J. Lyons

lyonstj@musc.edu

1 Centre for Experimental Medicine, Queen's University Belfast, Belfast, Northern Ireland, UK

2 Division of Endocrinology and Diabetes, CSB Suite 822, Medical University of South Carolina, Charleston, SC 29425, USA

3 The Department of Clinical Biochemistry, Royal Victoria Hospital, Belfast, Northern Ireland, UK

4 Section of Endocrinology, University of Oklahoma Health Sciences Center, Oklahoma City, OK, USA

5 University of Sydney, NHMRC Clinical Trials Centre, Camperdown, Sydney, NSW, Australia

6 Royal Women's Hospital, Melbourne, VIC, Australia
$12.4 \pm 1.8$ weeks; Visit 2, $21.7 \pm 1.4$ weeks; and Visit 3, $31.4 \pm 1.5$ weeks. All the women were free of microalbuminuria and hypertension at enrolment. All study visits preceded the clinical onset of pre-eclampsia.

Results In all groups, leptin, the ratio of leptin to total or HMW adiponectin, FABP4 concentration, ratio of FABP4 to total or HMW adiponectin and resistin level increased, while total and HMW adiponectin decreased, with gestational age. At Visit 1: (1) in diabetic women with vs without subsequent pre-eclampsia, leptin, ratio of leptin to total or HMW adiponectin, and ratio of FABP4 to total or HMW adiponectin, were increased $(p<0.05)$, while total adiponectin was decreased $(p<0.05)$; and (2) in normotensive diabetic vs nondiabetic women, total adiponectin was elevated $(p<0.05)$. At Visits 2 and 3: (1) the primary findings in the two diabetic groups persisted, and FABP4 also increased in women with subsequent pre-eclampsia $(p<0.05)$; and (2) there were no differences between the two normotensive groups. By logistic regression analyses after covariate adjustment $\left(\mathrm{HbA}_{1 \mathrm{c}}\right.$, insulin

Department of Endocrinology, Oslo University Hospital, Oslo, Norway

8 Institute of Clinical Medicine, University of Oslo, Oslo, Norway

9 Barbara Davis Center for Childhood Diabetes, University of Colorado, Denver, CO, USA

10 Department of Regenerative Medicine and Cell Biology, Medical University of South Carolina, Charleston, SC, USA

11 Spartanburg Regional Hospital, Spartanburg, SC, USA

12 Department of Pediatrics, University of Oklahoma Health Sciences Center, Oklahoma City, OK, USA

13 Centre for Public Health, Queen's University Belfast, Belfast, Northern Ireland, UK 
$\mathrm{kg}^{-1}$ day $^{-1}$ and gestational age), the best predictive models for pre-eclampsia were as follows: Visit 1, doubling of leptin, OR $9.0(p<0.01)$; Visit 2, doubling of the leptin:total adiponectin ratio, OR 3.7 ( $p<0.05)$; and Visit 3, doubling of FABP4 concentration, OR $25.1(p<0.01)$. The associations were independent of BMI.

Conclusions/interpretation As early as the first trimester in type 1 diabetic women, adipokine profiles that suggest insulin resistance are associated with subsequent pre-eclampsia, possibly reflecting maternal characteristics that precede pregnancy. These associations persist in the second and third trimesters, and are independent of BMI. Insulin resistance may predispose women with type 1 diabetes to pre-eclampsia.

Keywords Adipokine $\cdot$ Adiponectin $\cdot$ Diabetes $\cdot$ Fatty acid binding protein $\cdot$ Leptin $\cdot$ Pre-eclampsia $\cdot$ Pregnancy

$\begin{array}{ll}\text { Abbreviations } \\ \text { CRP } & \text { C-reactive protein } \\ \text { DM- } & \text { Non-diabetic normotensive (group) } \\ \text { DM+PE- } & \text { Type } 1 \text { diabetes mellitus, normotensive (group) } \\ \text { DM+PE+ } & \text { Type 1 diabetes mellitus, pre-eclampsia (group) } \\ \text { FABP4 } & \text { Fatty acid binding protein 4 } \\ \text { GDM } & \text { Gestational diabetes mellitus } \\ \text { HMW } & \text { High molecular weight (also known as high mo- } \\ & \text { lecular mass) } \\ \text { IDI } & \text { Integrated Discrimination Improvement } \\ \text { MAP } & \text { Mean arterial pressure } \\ \text { NPV } & \text { Negative predictive value } \\ \text { NRI } & \text { Net Reclassification Improvement } \\ \text { PlGF } & \text { Placental growth factor } \\ \text { PPV } & \text { Positive predictive value } \\ \text { RBP4 } & \text { Retinol binding protein } 4 \\ \text { ROC } & \text { Receiver operating characteristic } \\ \text { sFlt-1 } & \text { Soluble fms-like tyrosine kinase-1 }\end{array}$

\section{Introduction}

Pre-eclampsia is a leading cause of maternal and neonatal morbidity and mortality worldwide. It is a multisystem disorder characterised by a new onset, in the second half of pregnancy ( $\geq 20$ weeks' gestation), of hypertension accompanied by proteinuria and/or end-organ dysfunction [1-3]. In women with pregestational diabetes mellitus (either type 1 or type 2), the risk is increased about fourfold compared with nondiabetic women (around 20\% vs around 5\%) [4], and women with the metabolic syndrome are also at increased risk [5]. Insulin resistance is a hallmark of the metabolic syndrome and type 2 diabetes $[6,7]$ but also affects some individuals with type 1 diabetes [8-12]. Pregnancy itself induces insulin resistance, especially after 20 weeks' gestation $[13,14]$.
Hypertension, obesity, dyslipidaemia, systematic inflammation and long-term risk of cardiovascular and renal disease are associated with both insulin resistance and prior preeclampsia [2, 15-18]. It follows that, early in pregnancy and even in women with pregestational type 1 diabetes, maternal insulin resistance might be associated with the development of pre-eclampsia.

The measurement of insulin resistance is challenging. Convenient estimates (e.g. HOMA or QUICKI [14, 17, 19]) depend upon fasting insulin and glucose levels and cannot be used in individuals with type 1 diabetes who take exogenous insulin and in whom plasma glucose levels are highly variable. More precise estimates from 'insulin clamp' studies can be obtained in those with type 1 diabetes $[8,9]$, including during pregnancy [20], but this approach is too cumbersome for routine clinical use. Circulating adipokines may serve as indirect measures of insulin resistance in both pregnant and non-pregnant states $[5,13,21,22]$ and therefore merit investigation as candidate biomarkers for pre-eclampsia.

Adipokines have effects on insulin secretion, insulin action, energy expenditure, inflammation, regulation of adipogenesis and reproduction [23]. Adipose tissue is considered their main source, but in pregnancy they may also be produced by the placenta. Leptin modulates satiety, stimulates energy expenditure, and inhibits insulin secretion [24, 25]. It is proinflammatory, decreasing the production of anti-inflammatory adiponectin. Adiponectin (total and high molecular weight [HMW; also known as high molecular mass] forms) stimulates fatty acid oxidation, decreases plasma triacylglycerols and increases insulin sensitivity [26-29]. Fatty acid binding protein 4 (FABP4) integrates metabolic and inflammatory pathways, is implicated in insulin resistance and atherosclerosis $[30,31]$ and is expressed in macrophages and placenta in addition to adipose tissue [32]. Resistin is secreted by macrophages, monocytes and white adipose tissue; it impairs glucose uptake by adipocytes, raises plasma glucose and promotes inflammation and insulin resistance [33]. Retinol binding protein 4 (RPB4) is involved in vitamin A (retinol) transport; it is secreted by the liver and adipose tissue and increases systemic insulin resistance [7, 34, 35].

Using samples from a prospective study of pregnancy in women with type 1 diabetes [36,37], we measured these adipokines (and ratios of those mediating insulin resistance vs sensitivity) in the first, second and early third trimesters, comparing women who did with those who did not develop pre-eclampsia (primary analysis). We also included a group of normotensive, non-diabetic women to provide reference values, and compared normotensive women with and without type 1 diabetes (secondary analysis). No previous study has evaluated this group of adipokines throughout gestation as potential predictors of pre-eclampsia in women with type 1 diabetes. 


\section{Methods}

Study design and participants Study participants were selected from a previously described prospective cohort of 151 women with type 1 diabetes and 24 non-diabetic women studied longitudinally throughout pregnancy [36]. The study was approved by the Institutional Review Boards of all the participating institutions and by the Ethics Committee of the School of Medicine, Dentistry and Biomedical Sciences, Queen's University Belfast; it was conducted according to the principles of the Declaration of Helsinki.

Clinical data and specimens were collected at three study visits (means $\pm \mathrm{SD}$ ): Visit $1,12.4 \pm 1.8$ weeks; Visit 2, $21.7 \pm 1.4$ weeks; and Visit $3,31.4 \pm 1.5$ weeks of gestation (no overlap); in women with pre-eclampsia, Visit 3 preceded its clinical onset. Blood and urine samples were obtained after an overnight fast and in participants with type 1 diabetes, prior to insulin administration. Women with renal impairment (including microalbuminuria), cardiovascular disease, hypertension or other significant medical problems, either before pregnancy or at Visit 1, were excluded. Pre-eclampsia was defined as new-onset hypertension (>140/90 mmHg) after 20 weeks' gestation accompanied by proteinuria $(>300 \mathrm{mg} / 24 \mathrm{~h}$ ) in a previously normotensive woman. Groups were defined as follows: $\mathrm{DM}+\mathrm{PE}+$, type 1 diabetic women who developed preeclampsia; $\mathrm{DM}+\mathrm{PE}-$, type 1 diabetic women who remained normotensive and normo-albuminuric; and DM-, nondiabetic women who remained normotensive.

From the larger cohort, we previously selected (for our primary comparison and as per the original study design) a subset comprising all $26 \mathrm{DM}+\mathrm{PE}+$ women and a matched group of $26 \mathrm{DM}+\mathrm{PE}-$ individuals (matched by age, duration of diabetes, $\mathrm{HbA}_{1 \mathrm{c}}$ and parity). For a secondary analysis, we compared the 26 normotensive diabetic women with all those available who did not have diabetes or pre-eclampsia (DM$n=21$ ). For the present study, sample attrition reduced these numbers, but we included all women from these groups for whom samples were available, i.e. $23 \mathrm{DM}+\mathrm{PE}+, 24 \mathrm{DM}+\mathrm{PE}-$ and $19 \mathrm{DM}-$ women.

Laboratory measures Plasma leptin, HMW adiponectin, FABP4, resistin and RBP4 were measured by Quantikine ELISA kits (R\&D Systems, Minneapolis, MN, USA) as per the manufacturer's instructions. Plasma total adiponectin was measured using a DuoSet ELISA kit (R\&D Systems), as per the manufacturer's instructions. From the time of collection, all samples were stored at $-80^{\circ} \mathrm{C}$ until analysis. In assay validation, each variable was stable through multiple freeze/ thaw cycles. Average intra- and interassay CV for internal controls for all assays were $6 \%$ and $7 \%$, respectively. All assays were performed in duplicate using kits with the same batch number, by operators blinded to sample identity.
Statistical analysis Normally distributed continuous variables were summarised as means (SD or 95\% CI). Non-normally distributed variables were log-transformed and expressed as geometric means (95\% CI of geometric mean); these were for leptin, leptin:adiponectin (total and HMW) ratio, FABP4, FABP4:adiponectin (total and HMW) ratio, HMW adiponectin and resistin. Primary analyses compared the $\mathrm{DM}+\mathrm{PE}+$ and $\mathrm{DM}+\mathrm{PE}-$ groups; secondary analyses compared the $\mathrm{DM}+\mathrm{PE}-$ and $\mathrm{DM}-$ groups.

Group comparisons were analysed using unpaired Student's $t$ tests or Mann-Whitney tests (for continuous variables) or $\chi^{2}$ tests (for categorical variables). Analyses of repeated measures used Friedman's test. Logistic regression was used to assess associations of selected adipokines with risk of pre-eclampsia in women with type 1 diabetes (DM- groups were excluded from the regression models), with and without adjustment for contemporaneous $\mathrm{HbA}_{1 \mathrm{c}}$, insulin dose (total and per kg per day), gestational age, and BMI (factors selected according to baseline differences, known associations with pre-eclampsia or both) at blood sampling. Including all variables selected for matching had no effect on the conclusions, so only selected covariates were included. As leptin, leptin:adiponectin ratio, FABP4 and FABP4:adiponectin ratio were not normally distributed but showed a positive skew, values were logarithmically transformed (base 2) before inclusion in any regression model. The predictive ability of logistic regression was assessed from the sensitivity, specificity, positive predictive value (PPV) and negative predictive value (NPV) for the predicted probability of pre-eclampsia. Due to the oversampling of the participants with pre-eclampsia in this subset, the PPV and NPV were reported after adjusting the constant in the logistic regression. To assess the performance of candidate biomarkers at each trimester, a receiver operating characteristic (ROC) curve analysis was performed using the predicted probabilities from logistic regression analyses, with and without covariates, and the increase in area under the ROC curve on addition of each individual biomarker was tested [38].

The category-free Net Reclassification Improvement (NRI) index and the Integrated Discrimination Improvement (IDI) index were calculated to quantify the additional clinical value of a biomarker over established clinical risk factors alone [39, 40]. The NRI and IDI indices compare the predicted probabilities of pre-eclampsia obtained from the logistic model of clinical risk factors before and after the addition of each biomarker. The NRI index is the net proportion of probabilities increased for women who developed preeclampsia, and decreased for women without pre-eclampsia, resulting from the addition of a biomarker to the logistic regression model [40]. The IDI index is defined as the average increase in predicted risk in women with pre-eclampsia, added to the average decrease in predicted risk in women without pre-eclampsia, due to the addition of a biomarker to the logistic model [39]. Statistical significance was defined as 
$p<0.05$ (two-sided). Statistical analyses were performed using SPSS software, version 22 (IBM Corp, Armonk, NY, USA), Stata Release 13 (StataCorp, College Station, TX, USA) and statistical software package R (R-3.4.1; Foundation for Statistical Computing, Vienna, Austria).

\section{Results}

Clinical characteristics Maternal characteristics at baseline (Visit 1) are shown in Table 1. Between the DM+PE+ and $\mathrm{DM}+\mathrm{PE}-$ groups, there were no significant differences in age, gestational age, gravida, parity, number of miscarriages, age at onset of diabetes, diabetes duration, BP, mean arterial pressure (MAP), total cholesterol, LDL-cholesterol, triacylglycerol level, C-reactive protein (CRP), or microalbumin:creatinine ratio. Women in the $\mathrm{DM}+\mathrm{PE}+$ group had significantly higher $\mathrm{BMI}, \mathrm{HbA}_{1 \mathrm{c}}$, total daily insulin requirements, insulin dose per kilogram and eGFR, and lower HDL-cholesterol, than those in the $\mathrm{DM}+\mathrm{PE}-$ group (all $p<0.05)$. Women in the $\mathrm{DM}+\mathrm{PE}+$ group were delivered earlier than those in the DM+PE- group $(p<0.05)$.

Most maternal characteristics did not differ between the $\mathrm{DM}+\mathrm{PE}-$ and $\mathrm{DM}-$ groups, but diabetic women had, as expected, higher $\mathrm{HbA}_{1 \mathrm{c}}$ levels $(p<0.0001)$ and earlier delivery $(p<0.05)$.

Leptin In the $\mathrm{DM}+\mathrm{PE}+$ vs the $\mathrm{DM}+\mathrm{PE}-$ group, leptin was increased throughout gestation: Visit $1, p<0.01$; Visit 2, $p<0.01$; Visit $3, p<0.05$ (Table 2). There were no significant differences in leptin at any time point between the DM+PEand DM- groups. Leptin increased throughout pregnancy in both normotensive groups (both $p<0.01$ ), but not significantly in the $\mathrm{DM}+\mathrm{PE}+$ group.

Leptin:adiponectin ratio These adipokines have opposite effects on insulin resistance, and their ratio is therefore of interest. In the $\mathrm{DM}+\mathrm{PE}+\mathrm{vs} \mathrm{DM}+\mathrm{PE}-$ group, the leptin:total adiponectin ratio and the leptin:HMW adiponectin ratio were both elevated at Visits 1 and $2(p<0.01)$. The ratios did not differ between the two normotensive groups. Leptin:total adiponectin ratio and leptin:HMW adiponectin ratio increased progressively throughout pregnancy $(p<0.01$ and $p<0.001$, respectively) in all three groups. Since the findings for these two ratios were similar, only the leptin:total adiponectin ratio was used in further analyses.

FABP4 In the DM+PE+ vs the DM+PE- group-, FABP4 was increased at Visit $2(p<0.01)$ and Visit $3(p<0.001)$ (Table 2). FABP4 did not differ at any time point between the two normotensive groups. FABP4 increased progressively throughout pregnancy in all three groups $(p \leq 0.001)$.
FABP4:adiponectin ratio In the $\mathrm{DM}+\mathrm{PE}+\mathrm{vs}$ the $\mathrm{DM}+\mathrm{PE}-$ group, FABP4:total adiponectin and FABP4:HMW adiponectin ratios were elevated at all study visits (Visit 1 , $p<0.05$; Visit $2, p<0.01$; Visit $3, p<0.05$ ). They did not differ at any time point between the DM+PE- and DMgroups. Both ratios increased progressively throughout pregnancy in all three groups $(p<0.001)$. The results were similar for the two ratios; therefore only FABP4:total adiponectin was used for the further analyses.

Total adiponectin In the $\mathrm{DM}+\mathrm{PE}+\mathrm{vs}$ the $\mathrm{DM}+\mathrm{PE}-$ group, total adiponectin was lower at Visit $1(p<0.05)$ (Table 2). In addition, total adiponectin was higher at Visit 1 in the $\mathrm{DM}+\mathrm{PE}-$ than the $\mathrm{DM}-$ group $(p=0.01)$. Total adiponectin decreased progressively throughout pregnancy in all groups $(\mathrm{DM}+\mathrm{PE}+, p<0.01 ; \mathrm{DM}+\mathrm{PE}-, p<0.001 ; \mathrm{DM}-, p<0.05)$.

HMW adiponectin In the $\mathrm{DM}+\mathrm{PE}+\mathrm{vs} \mathrm{DM}+\mathrm{PE}-$ group, HMW adiponectin was lower at Visit $1(p=0.05)$ and Visit $2(p<0.05)$ (Table 2). HMW adiponectin did not differ significantly between the normotensive groups at any visit. HMW adiponectin decreased progressively throughout pregnancy in all three groups $(\mathrm{DM}+\mathrm{PE}+, p<0.01$; DM+PE-, $p<0.001 ; \mathrm{DM}-, p<0.001)$.

Resistin Levels of resistin did not differ between any of the groups at any time point (Table 2) but increased progressively throughout pregnancy in all three groups $(\mathrm{DM}+\mathrm{PE}+, p<0.01$; $\mathrm{DM}+\mathrm{PE}-, p<0.05 ; \mathrm{DM}-, p<0.001)$.

RBP4 Levels of RBP4 did not differ between any of the groups at any time point (Table 2). In addition, they increased in all groups between Visit 1 and Visit $2(p<0.05)$. There were no significant changes between Visits 2 and 3.

Analyses controlling for relevant covariates To assess the effectiveness of adipokines as biomarkers for pre-eclampsia early in pregnancy, data were analysed by logistic regression with and without covariates $\left(\mathrm{HbA}_{1 \mathrm{c}}\right.$, insulin dose $\mathrm{kg}^{-1}$ day $^{-1}$ and gestational age) (Table 3). The adipokines identified above in our primary analyses (leptin, leptin:total adiponectin ratio, FABP4, FABP4:total adiponectin ratio and total adiponectin) were included individually and in combination.

Using all available data and after adjustment for $\mathrm{HbA}_{1 \mathrm{c}}$, insulin $\mathrm{kg}^{-1}$ day $^{-1}$ and gestational age, the best model indicated that, at Visit 1, a doubling of leptin was associated with an approximately ninefold higher odds of pre-eclampsia (OR 9.0 [2.0-40.3], $p=0.004$; AUC 0.902, $p=0.10$ vs established risk factors alone). The NRI and IDI indices showed that the addition of leptin to maternal factors $\left(\mathrm{HbA}_{1 \mathrm{c}}\right.$, insulin kg ${ }^{-1}$ day $^{-1}$ and gestational age) significantly increased the correct classification of diabetic women with and without pre-eclampsia, and increased the discrimination between pre-eclampsia and 
Table 1 Clinical characteristics at study entry of women with type 1 diabetes with and without pre-eclampsia, and of normotensive non-diabetic control participants

\begin{tabular}{|c|c|c|c|c|c|}
\hline Clinical characteristics & $\mathrm{DM}+\mathrm{PE}+(n=23)$ & $p$ value $^{\mathrm{a}}$ & $\mathrm{DM}+\mathrm{PE}-(n=24)$ & $p$ value $^{\mathrm{b}}$ & $\mathrm{DM}-(n=19)$ \\
\hline Age of woman (years) & $28.5 \pm 5.6$ & 0.31 & $29.9 \pm 3.8$ & 0.25 & $31.4 \pm 4.5$ \\
\hline BMI $\left(\mathrm{kg} / \mathrm{m}^{2}\right)$ & $27.9 \pm 5.9$ & 0.03 & $24.6 \pm 4.1$ & 0.50 & $23.8 \pm 3.8$ \\
\hline$\leq 25 \mathrm{~kg} / \mathrm{m}^{2}$ (normal) & $9(39.1)$ & 0.02 & $16(66.7)$ & 0.92 & $13(68.4)$ \\
\hline $25-30 \mathrm{~kg} / \mathrm{m}^{2}$ (overweight) & $4(17.4)$ & & $6(25.0)$ & & $5(26.3)$ \\
\hline$\geq 30 \mathrm{~kg} / \mathrm{m}^{2}$ (obese) & $10(43.5)$ & & $2(8.3)$ & & $1(5.3)$ \\
\hline Gravida & $1.3 \pm 0.7$ & 1.00 & $1.3 \pm 0.7$ & 0.19 & $1.7 \pm 1.0$ \\
\hline Para & $0.2 \pm 0.5$ & 0.91 & $0.2 \pm 0.5$ & 0.13 & $0.5 \pm 1.0$ \\
\hline Miscarriages & $0.1 \pm 0.4$ & 0.91 & $0.1 \pm 0.3$ & 0.81 & $0.2 \pm 0.4$ \\
\hline Age at onset of T1DM (years) & $11.5 \pm 5.5$ & 0.07 & $15.2 \pm 7.5$ & - & - \\
\hline Duration of T1DM (years) & $16.8 \pm 6.8$ & 0.32 & $14.8 \pm 7.0$ & - & - \\
\hline $\mathrm{HbA}_{1 \mathrm{c}}(\%)$ & $7.4 \pm 1.2$ & 0.05 & $6.7 \pm 1.0$ & $<0.0001$ & $5.3 \pm 0.3$ \\
\hline $\mathrm{HbA}_{1 \mathrm{c}}(\mathrm{mmol} / \mathrm{mol})$ & $57 \pm 14$ & 0.05 & $50 \pm 11$ & $<0.0001$ & $35 \pm 3$ \\
\hline \multicolumn{6}{|l|}{ Blood pressure (mmHg) } \\
\hline Systolic BP & $113.1 \pm 12.4$ & 0.26 & $109.4 \pm 9.6$ & 0.23 & $113.3 \pm 8.7$ \\
\hline Diastolic BP & $66.6 \pm 9.0$ & 0.27 & $63.8 \pm 8.1$ & 0.24 & $66.9 \pm 7.6$ \\
\hline MAP & $82.1 \pm 9.0$ & 0.21 & $79.0 \pm 7.7$ & 0.14 & $82.7 \pm 6.2$ \\
\hline Total daily insulin (units/day) & $62.2 \pm 19.7$ & 0.01 & $47.9 \pm 14.2$ & - & - \\
\hline Insulin (units/kg/day) & $0.81 \pm 0.14$ & 0.02 & $0.69 \pm 0.17$ & - & - \\
\hline Total cholesterol (mmol/l) & $4.7 \pm 0.7$ & 0.53 & $4.5 \pm 0.9$ & 0.18 & $4.9 \pm 0.7$ \\
\hline HDL-cholesterol (mmol/l) & $1.9 \pm 0.4$ & 0.03 & $2.2 \pm 0.5$ & 0.71 & $2.1 \pm 0.6$ \\
\hline LDL-cholesterol (mmol/1) & $2.4 \pm 0.7$ & 0.08 & $2.0 \pm 0.7$ & 0.18 & $2.3 \pm 0.8$ \\
\hline Triacylglycerols (mmol/l) & $1.0 \pm 0.3$ & 0.27 & $0.8 \pm 0.3$ & 0.09 & $1.1 \pm 0.4$ \\
\hline Urine microalbumin (mg/l) & $10.3 \pm 17.8$ & 0.12 & $4.3 \pm 2.0$ & 0.69 & $4.6 \pm 1.7$ \\
\hline Urine creatinine $(\mu \mathrm{mol} / \mathrm{l})$ & $6.5 \pm 3.8$ & 0.99 & $6.5 \pm 2.7$ & 0.73 & $6.2 \pm 3.0$ \\
\hline Microalbumin:creatinine ratio & $0.7(0.51 .2)$ & 0.62 & $0.7(0.41 .0)$ & 0.40 & $0.8(0.61 .0)$ \\
\hline eGFR $\left(\mathrm{ml} \mathrm{min} \min ^{-1} 1.73 \mathrm{~m}^{-2}\right)$ & $125.0 \pm 7.7$ & 0.02 & $119.4 \pm 7.0$ & 0.92 & $119.7 \pm 8.3$ \\
\hline CRP (nmol/l) & $68.5 \pm 67.0$ & 0.26 & $45.9 \pm 68.3$ & 0.55 & $62.1 \pm 100.8$ \\
\hline \multicolumn{6}{|l|}{ Gestational age (weeks) } \\
\hline Visit 1 & $12.3 \pm 2.1$ & 0.94 & $12.3 \pm 1.7$ & 0.49 & $12.6 \pm 1.7$ \\
\hline Visit 2 & $22.1 \pm 1.6$ & 0.18 & $21.5 \pm 1.3$ & 0.95 & $21.5 \pm 1.3$ \\
\hline Visit 3 & $31.7 \pm 1.7$ & 0.39 & $31.3 \pm 1.5$ & 0.82 & $31.2 \pm 1.1$ \\
\hline Delivery & $37.0 \pm 1.3$ & 0.01 & $38.0 \pm 1.4$ & 0.01 & $39.2 \pm 1.6$ \\
\hline
\end{tabular}

Data are presented as means $\pm \mathrm{SD}, n(\%)$ or median (interquartile range). Measurements refer to Visit 1 unless otherwise indicated. Independent samples $t$ tests, Mann-Whitney tests and $\chi^{2}$ tests were used as appropriate

eGFR was defined using the Chronic Kidney Disease Epidemiology Collaboration equation [CKD-EPI]

$p$ values $<0.05$ (statistically significant)

${ }^{\mathrm{a}} p$ value, $\mathrm{DM}+\mathrm{PE}+\mathrm{vs} \mathrm{DM}+\mathrm{PE}-$

${ }^{\mathrm{b}} p$ value, $\mathrm{DM}+\mathrm{PE}-\mathrm{vs} \mathrm{DM}-$

T1DM, type 1 diabetes mellitus

non-pre-eclampsia in women at Visit 1 (NRI, $p=0.004$; IDI, $p<0.001)$. The final model resulted in a sensitivity of $81 \%$ $(17 / 21)$ and a specificity of $80 \%(16 / 20)$. Adjustment to account for the $21 \%$ prevalence of pre-eclampsia in the overall cohort yielded a PPV of $100 \%$ and an NPV of $69 \%$.

At Visit 2, the best model indicated that a doubling of leptin:total adiponectin ratio was associated with an approximately fourfold higher odds of pre-eclampsia (OR $3.7[1.3$,
10.7], $p=0.014$; AUC 0.787, $p=0.088$ vs established risk factors alone). The NRI and IDI indices showed that the addition of leptin:total adiponectin ratio improved the classification and discrimination of women with and without pre-eclampsia at Visit 2 (NRI, $p=0.03$; IDI, $p=0.01$ ). The final model resulted in a sensitivity of $84 \%(16 / 19)$ and a specificity of $68 \%(13 / 19)$. Adjusting for pre-eclampsia prevalence yielded a PPV of $100 \%$ and an NPV of $56 \%$. 
Table 2 Levels of circulating adipokine profile prior to onset of pre-eclampsia

\begin{tabular}{|c|c|c|c|c|c|}
\hline Variable & $\mathrm{DM}+\mathrm{PE}+(n=23)$ & $p$ value $^{\mathrm{a}}$ & $\mathrm{DM}+\mathrm{PE}-(n=24)$ & $p$ value $^{\mathrm{b}}$ & $\mathrm{DM}-(n=19)$ \\
\hline \multicolumn{6}{|c|}{$\operatorname{Leptin}^{\mathrm{c}}(\mu \mathrm{g} / \mathrm{l})$} \\
\hline Visit 1 & $27.7(21.8,35.1)$ & 0.003 & $16.3(12.7,20.9)$ & 0.74 & $17.3(12.5,24.2)$ \\
\hline Visit 2 & $34.9(29.2,41.7)$ & 0.004 & $23.3(18.8,28.8)$ & 0.71 & $21.6(14.8,31.5)$ \\
\hline Visit 3 & $36.6(28.0,47.8)$ & 0.03 & $24.6(19.4,31.1)$ & 0.71 & $26.4(18.7,37.3)$ \\
\hline \multicolumn{6}{|c|}{ Leptin:total adiponectin ratio ${ }^{c}$} \\
\hline Visit 1 & $3.8(2.8,5.2)$ & 0.003 & $1.9(1.4,2.7)$ & 0.19 & $2.6(1.8,3.8)$ \\
\hline Visit 2 & $5.5(4.5,6.7)$ & 0.004 & $3.1(2.2,4.3)$ & 0.99 & $3.1(2.1,4.7)$ \\
\hline Visit 3 & $5.7(4.2,7.8)$ & 0.09 & $3.8(2.7,5.5)$ & 0.64 & $4.4(2.9,6.7)$ \\
\hline \multicolumn{6}{|c|}{ Leptin:HMW adiponectin ratio ${ }^{c}$} \\
\hline Visit 1 & $3.5(2.4,5.2)$ & 0.004 & $1.5(1.0,2.3)$ & 0.38 & $2.0(1.3,3.1)$ \\
\hline Visit 2 & $5.4(3.9,7.5)$ & 0.005 & $2.6(1.8,3.9)$ & 0.96 & $2.7(1.6,4.3)$ \\
\hline Visit 3 & $5.8(3.9,8.8)$ & 0.06 & $3.5(2.4,5.1)$ & 0.80 & $4.4(2.8,6.9)$ \\
\hline \multicolumn{6}{|c|}{$\mathrm{FABP}^{\mathrm{c}}(\mathrm{ng} / \mathrm{ml})$} \\
\hline Visit 1 & $9.4(7.9,11.1)$ & 0.07 & $7.6(6.5,9.0)$ & 0.24 & $6.6(5.3,8.1)$ \\
\hline Visit 2 & $11.1(9.4,13.1)$ & 0.005 & $8.0(6.8,9.3)$ & 0.41 & $7.3(6.1,8.7)$ \\
\hline Visit 3 & $15.6(13.4,18.1)$ & $<0.001$ & $10.2(8.8,11.7)$ & 0.36 & $9.1(7.4,11.2)$ \\
\hline \multicolumn{6}{|c|}{ FABP4:total adiponectin ratio $^{c}$} \\
\hline Visit 1 & $1.3(1.0,1.7)$ & 0.03 & $0.9(0.7,1.1)$ & 0.54 & $1.0(0.8,1.2)$ \\
\hline Visit 2 & $1.7(1.4,2.2)$ & 0.004 & $1.1(0.9,1.3)$ & 0.91 & $1.1(0.9,1.3)$ \\
\hline Visit 3 & $2.4(2.0,3.0)$ & 0.01 & $1.6(1.2,2.1)$ & 0.77 & $1.5(1.1,2.1)$ \\
\hline \multicolumn{6}{|c|}{ FABP4:HMW adiponectin ratio ${ }^{c}$} \\
\hline Visit 1 & $1.2(0.8,1.8)$ & 0.02 & $0.7(0.5,1.0)$ & 0.99 & $0.7(0.6,0.9)$ \\
\hline Visit 2 & $1.7(1.2,2.4)$ & 0.005 & $0.9(0.7,1.2)$ & 0.97 & $0.9(0.7,1.1)$ \\
\hline Visit 3 & $2.5(1.9,3.3)$ & 0.01 & $1.4(1.1,2.0)$ & 0.84 & $1.5(1.1,2.1)$ \\
\hline \multicolumn{6}{|c|}{ Total adiponectin $(\mu \mathrm{g} / \mathrm{ml})$} \\
\hline Visit 1 & $7.5(6.7,8.3)$ & 0.04 & $8.8(7.8,9.8)$ & 0.01 & $6.9(5.9,8.0)$ \\
\hline Visit 2 & $6.6(5.7,7.6)$ & 0.09 & $7.9(6.7,9.0)$ & 0.21 & $7.0(6.3,7.7)$ \\
\hline Visit 3 & $6.5(5.8,7.2)$ & 0.65 & $6.8(5.8,7.9)$ & 0.44 & $6.3(5.4,7.2)$ \\
\hline \multicolumn{6}{|c|}{ HMW adiponectin ${ }^{\mathrm{c}}(\mu \mathrm{g} / \mathrm{ml})$} \\
\hline Visit 1 & $7.9(6.3,9.9)$ & 0.05 & $10.5(8.6,12.9)$ & 0.28 & $9.2(7.711 .1)$ \\
\hline Visit 2 & $6.4(5.1,8.0)$ & 0.04 & $8.8(7.0,11.1)$ & 0.43 & $8.1(6.6,9.9)$ \\
\hline Visit 3 & 6.3. $(5.0,7.9)$ & 0.44 & $7.0(5.7,8.7)$ & 0.45 & $6.0(5.0,7.3)$ \\
\hline \multicolumn{6}{|c|}{ HMW:total adiponectin } \\
\hline Visit 1 & $1.2(1.0,1.4)$ & 0.33 & $1.3(1.1,1.4)$ & 0.52 & $1.3(1.2,1.5)$ \\
\hline Visit 2 & $1.1(0.9,1.3)$ & 0.29 & $1.2(1.1,1.4)$ & 0.99 & $1.2(1.0,1.4)$ \\
\hline Visit 3 & $1.1(0.9,1.3)$ & 0.36 & $1.2(0.9,1.5)$ & 0.52 & $1.1(0.9,1.2)$ \\
\hline \multicolumn{6}{|c|}{$\operatorname{Resistin}^{\mathrm{c}}$ (ng/ml) } \\
\hline Visit 1 & $9.5(8.1,11.1)$ & 0.93 & $9.4(8.1,10.9)$ & 0.47 & $8.7(7.9,9.7)$ \\
\hline Visit 2 & $10.8(9.4,12.3)$ & 0.39 & $9.9(8.6,11.5)$ & 0.66 & $9.5(8.5,10.7)$ \\
\hline Visit 3 & $11.2(9.6,13.0)$ & 0.61 & $10.7(9.4,12.0)$ & 0.40 & $9.9(8.8,11.2)$ \\
\hline \multicolumn{6}{|c|}{ RBP4 $(\mu \mathrm{g} / \mathrm{ml})$} \\
\hline Visit 1 & $21.2(19.7,22.6)$ & 0.89 & $21.4(18.1,24.7)$ & 0.36 & $23.3(21.4,25.1)$ \\
\hline Visit 2 & $23.6(21.3,25.8)$ & 0.77 & $23.1(20.4,25.7)$ & 0.07 & $26.0(24.1,28.0)$ \\
\hline Visit 3 & $22.0(19.3,24.7)$ & 0.91 & $22.2(19.7,24.7)$ & 0.12 & $24.9(22.5,27.2)$ \\
\hline
\end{tabular}

$p$ values are reported from parametric or log-transformed analyses as appropriate. $p$ values $<0.05$ (statistically significant)

${ }^{\mathrm{a}} p$ value, $\mathrm{DM}+\mathrm{PE}+\mathrm{vs} \mathrm{DM}+\mathrm{PE}-$

${ }^{\mathrm{b}} p$ value, $\mathrm{DM}+\mathrm{PE}-\mathrm{vs} \mathrm{DM}-$

${ }^{\mathrm{c}}$ Geometric mean (95\% CI of geometric mean); otherwise mean (95\% CI of mean) 
At Visit 3, the best model indicated that a doubling of FABP4 was associated with an approximately 25 -fold greater risk of pre-eclampsia (OR 25.1 [2.3, 273.8], $p=0.008$ ); AUC $0.847, p=0.1$ vs established risk factors alone). The NRI and IDI indices showed that the addition of FABP4 improved the classification and discrimination of women with and without pre-eclampsia at Visit 3 (NRI, $p<0.001$; IDI, $p=0.002$ ). The final model resulted in a sensitivity of $71 \%(12 / 17)$ and a specificity of $75 \%$ (15/20). Adjusting for the prevalence of pre-eclampsia yielded a PPV of $100 \%$ and an NPV of $65 \%$.

The strength of the associations with pre-eclampsia were not further improved by the inclusion of any two (or more) individual biomarkers at any time point.

Although the covariates shown in Table 3 take body weight into account, adipokines are known to be associated with BMI, and therefore we also analysed their associations with pre-eclampsia controlling for BMI alone, and for BMI combined with $\mathrm{HbA}_{1 \mathrm{c}}$, insulin dose and gestational age. At each study visit, BMI was significantly higher (by $t$ test) in diabetic women with vs without pre-eclampsia $(p<0.05$ at Visit 1 and Visit 2; $p<0.01$ at Visit 3), and at Visit 1 , a one unit increase in BMI raised the risk of pre-eclampsia by $15 \%$ (data not shown). However, when $\mathrm{HbA}_{1 \mathrm{c}}$, insulin dose (either daily total or per kilogram) and gestational age were taken into account, the significant associations between BMI and preeclampsia were lost (in contrast to the findings with adipokines, reported above). Furthermore, addition of BMI to individual adipokines did not improve the prediction of pre-eclampsia by IDI or NRI analyses at any study visit. We also tested the strength of the associations of leptin, leptin:adiponectin ratio and FABP4 with subsequent preeclampsia when BMI was considered as a sole covariate, and when it was combined in a covariate model with $\mathrm{HbA}_{1 \mathrm{c}}$, insulin dose (either daily dose or per kilogram per day), and gestational age. These analyses confirmed that leptin at Visit 1 $(p<0.01)$, leptin:adiponectin ratio at Visit $2(p<0.05)$ and FABP4 at Visit $3(p<0.05)$ were independently associated with the development of pre-eclampsia.

\section{Discussion}

This is the first prospective cohort study to assess the potential, individually and combined, of a group of adipokines as predictors of pre-eclampsia in women with type 1 diabetes. Women with type 1 diabetes have often been excluded from

Table 3 Odds ratios (OR) for the development of PE, area under the ROC curve and IDI and NRI indices for selected adipokines in a predictive model per study visit (with and without adjustment of covariates)

\begin{tabular}{|c|c|c|c|c|c|c|}
\hline Variable & $\begin{array}{l}\text { Unadjusted } \\
\text { OR }(95 \% \text { CI) }\end{array}$ & $\begin{array}{l}\text { Adjusted } \\
\text { OR }(95 \% \mathrm{CI})^{\mathrm{a}}\end{array}$ & $\begin{array}{l}\text { Area under the ROC } \\
\text { curve without covariates } \\
\left(p \text { value }^{\mathrm{b}}\right)\end{array}$ & $\begin{array}{l}\text { Area under the ROC } \\
\text { curve with covariates } \\
\left(p \text { value }{ }^{\mathrm{c}}\right)\end{array}$ & IDI ( $p$ value) & NRI ( $p$ value) \\
\hline \multicolumn{7}{|l|}{ Leptin $(\mu \mathrm{g} / \mathrm{l})$} \\
\hline Visit 1 & $3.2(1.4,7.5)$ & $9.0(2.0,40.3)$ & $0.747(0.004)$ & $0.902(0.10)$ & $0.250(<0.001)$ & $0.824(0.004)$ \\
\hline Visit 2 & $4.4(1.5,13.4)$ & $4.9(1.4,18.0)$ & $0.766(0.003)$ & $0.776(0.15)$ & $0.161(0.01)$ & $0.553(0.07)$ \\
\hline Visit 3 & $2.2(1.1,4.7)$ & $4.0(1.2,13.6)$ & $0.697(0.02)$ & $0.803(0.26)$ & $0.139(0.03)$ & $0.947(<0.001)$ \\
\hline \multicolumn{7}{|c|}{ Leptin:total adiponectin } \\
\hline Visit 1 & $2.4(1.3,4.7)$ & $3.5(1.4,8.8)$ & $0.747(0.004)$ & $0.871(0.20)$ & $0.177(0.008)$ & $0.819(0.003)$ \\
\hline Visit 2 & $2.9(1.3,6.7)$ & $3.7(1.3,10.7)$ & $0.766(0.003)$ & $0.787(0.09)$ & $0.171(0.01)$ & $0.632(0.03)$ \\
\hline Visit 3 & $1.6(0.9,2.7)$ & $3.0(1.1,8.2)$ & $0.667(0.05)$ & $0.797(0.22)$ & $0.119(0.04)$ & $1.029(<0.001)$ \\
\hline \multicolumn{7}{|c|}{ FABP4 (ng/ml) } \\
\hline Visit 1 & $2.7(0.9,8.3)$ & $5.5(1.0,29.8)$ & $0.654(0.07)$ & $0.810(0.64)$ & $0.077(0.11)$ & $0.733(0.01)$ \\
\hline Visit 2 & $5.4(1.5,19.5)$ & $6.6(1.4,30.7)$ & $0.737(0.007)$ & $0.766(0.17)$ & $0.144(0.02)$ & $0.663(0.03)$ \\
\hline Visit 3 & $10.7(2.6,44.4)$ & $25.1(2.3,273.8)$ & $0.806(<0.001)$ & $0.847(0.10)$ & $0.236(0.002)$ & $1.247(<0.001)$ \\
\hline \multicolumn{7}{|c|}{ FABP4:total adiponectin } \\
\hline Visit 1 & $2.4(1.1,5.5)$ & $2.9(1.0,8.5)$ & $0.684(0.03)$ & $0.819(0.52)$ & $0.07(0.13)$ & $0.729(0.01)$ \\
\hline Visit 2 & $3.7(1.4,10.0)$ & $4.9(1.4,17.9)$ & $0.751(0.005)$ & $0.790(0.07)$ & $0.165(0.01)$ & $0.737(0.01)$ \\
\hline Visit 3 & $2.6(1.2,5.9)$ & $6.9(1.2,39.4)$ & $0.707(0.02)$ & $0.809(0.20)$ & $0.153(0.02)$ & $0.794(0.009)$ \\
\hline
\end{tabular}

Leptin, leptin:total adiponectin ratio, FABP4 and FABP4:total adiponectin ratio were all logarithmically transformed to base 2 . Therefore the OR corresponds to a doubling of level

$p$ values $<0.05$ (statistically significant)

${ }^{\mathrm{a}}$ Adjusted for $\mathrm{HbA}_{1 \mathrm{c}}$, insulin per kilogram per day and gestational age per visit

${ }^{\mathrm{b}}$ Relative to AUC of 0.5 , for a logistic model containing only selected biomarker at the current visit

${ }^{\mathrm{c}}$ Relative to AUC of $0.786,0.640$ and 0.715 for a logistic model containing covariates only per visit, respectively 
previous studies seeking biomarkers and mechanisms for preeclampsia, despite being at unusually high risk. We found that, compared with those who remained normotensive, type 1 diabetic women with subsequent pre-eclampsia exhibited elevated plasma leptin at all time points studied; importantly therefore, leptin exhibited significant elevation as early as the first trimester (Visit 1). Also at Visit 1, the addition of leptin to a panel of established risk factors significantly improved the prediction of pre-eclampsia. At Visit 1 and Visit 2 leptin:adiponectin ratios were elevated in women with type 1 diabetes who later developed pre-eclampsia. FABP4 was an effective predictor slightly later than leptin, being significantly elevated in type 1 diabetic women with subsequent preeclampsia at Visits 2 and 3, while the FABP4:adiponectin ratio became predictive at Visit 3. The ratios of leptin and FABP4 to adiponectin are of interest because they may reflect the balance between pro- and anti-inflammatory adipokines. The associations were independent of relevant covariates, including BMI. In other studies of cardiovascular disease, these ratios have predicted events better than the individual measures [41, 42]. In the present study, they did not improve upon individual measures until later in pregnancy.

Although our data need confirmation, they suggest new biomarkers for pre-eclampsia that may be useful very early in pregnancy for women with type 1 diabetes, long before currently recognised biomarkers are effective. Improved prediction would have immediate benefit for the planning of antenatal care; it would also be key for the future conduct of studies that require risk stratification, selecting women at the highest risk to receive new interventions and minimising the number receiving unnecessary treatment.

To assess the potential 'real-world' clinical utility of the candidate biomarkers for pre-eclampsia, we analysed our findings at each trimester separately using logistic regression and the NRI and IDI indices. We showed that the addition of adipokine data (optimally leptin at Visit 1, leptin:total adiponectin ratio at Visit 2, and FABP4 at Visit 3) to established maternal risk factors significantly improved the prediction of pre-eclampsia in women with type 1 diabetes. A model including two or more adipokines did not provide any further improvement. In previous studies of this cohort, we reported that an imbalance of angiogenic factors (increased soluble fmslike tyrosine kinase-1 [sFlt-1], reduced placental growth factor [PlGF], elevated sFlt-1:PlGF ratio) could predict pre-eclampsia in women with type 1 diabetes at Visit 3 [36]. The present findings are important because they suggest that adipokines may enable an assessment of pre-eclampsia risk in women with type 1 diabetes much earlier in pregnancy.

Measures of leptin have not previously been reported in pregnancies complicated by both type 1 diabetes and preeclampsia. However, previous studies of non-diabetic women, mostly employing a single time point, have demonstrated associations between elevated maternal leptin, as early as 13 weeks' gestation, and the later clinical onset of pre-eclampsia or gestational diabetes mellitus [GDM] [5, 25, 29, 42, 43]. Our data are consistent with these, showing that in women with type 1 diabetes who later develop pre-eclampsia, circulating leptin, leptin:total adiponectin ratio and leptin:HMW adiponectin ratio were elevated early in pregnancy and remained so throughout. Maternal leptin levels are known to increase during normal pregnancy, peaking at 20-30 weeks of gestation and decreasing after birth [5, 25, 29, 42, 44]. Lekva et al [42] have suggested that a high leptin:adiponectin ratio in pregnancy complicated by GDM might be used to predict unfavourable cardiovascular risk in a 5-year follow up, being superior to either leptin or adiponectin alone. This suggests that that higher levels of leptin and/or leptin:adiponectin ratio at the beginning of pregnancy may have a role in the development of pre-eclampsia and/or future cardiovascular risk.

FABP4 has previously been studied as a predictor of preeclampsia both in type 1 diabetic women [45] and in nondiabetic women [30]. Our results are consistent with, but add to, the findings of those studies. As previously shown [45], FABP4 was elevated early in pregnancy in women with type 1 diabetes who later developed pre-eclampsia. In addition to this, the current study suggests that FABP4 predicts preeclampsia at Visit 3 and, at this time point does so more effectively than any other individual adipokine. It is established that the second half of pregnancy is a state of physiological insulin resistance [13, 14, 46, 47], but these findings suggest that more marked insulin resistance is associated with preeclampsia. Finally, the ratio of FABP4 to adiponectin was predictive of pre-eclampsia at all three time points.

In contrast to the other adipokines, plasma adiponectin is inversely correlated with insulin resistance, and normally decreases as pregnancy advances and insulin resistance increases [5, 27-29, 42]. Studies assessing adiponectin in the prediction of pre-eclampsia have shown conflicting results [5, 27-29, 48]. Nien et al [27] and Fasshauer et al [28] reported, in separate case-control studies in non-diabetic populations, that increased total adiponectin and/or HMW adiponectin late in pregnancy (around 30 weeks' gestation) is associated with a poor preeclampsia outcome. However, consistent with generally accepted beneficial associations of high adiponectin levels, and in agreement with our data, other studies have observed significantly lower concentrations at the beginning of pregnancy in non-diabetic women who subsequently developed preeclampsia $[29,48]$. Also in several studies, plasma adiponectin was significantly lower in women with GDM than in women without [5, 29, 42]. Randeva et al [26] reported that adiponectin levels were higher throughout pregnancy, and 9 months postpartum, in type 1 diabetic vs non-diabetic women (none of the women had any evidence of renal disease or hypertension).

We found no differences in the levels of maternal RBP4 or resistin between uncomplicated non-diabetic pregnancies, pregnancies in women with type 1 diabetes and pregnancies in those 
with type 1 diabetes and pre-eclampsia. Plasma levels of both adipokines increased in all groups between the first and second trimesters; resistin continued to increase between the second and third trimesters, while RBP4 decreased slightly in the second half of pregnancy. RBP4 and resistin are both implicated in insulin resistance and glucose metabolism [33-35]; the lack of any signal associated with pre-eclampsia was therefore somewhat surprising. However, the literature is also conflicting, with reports showing an increase, a decrease or no change in RBP4 and resistin concentrations in associations with complications of pregnancy [5, 22, 29, 47-49]. Thus, although RBP4 and resistin may show associations for obesity, insulin resistance/sensitivity and type 2 diabetes with cardiovascular disease [7, 33-35], the mixed data from pregnancy studies, and our current results, suggest that neither RBP4 nor resistin is an effective early predictor of pre-eclampsia.

The concept that relative insulin resistance early in type 1 diabetic pregnancy is associated with subsequent preeclampsia is consistent with a recent paper showing that impaired vasodilatory capacity, endothelial dysfunction and vasoactive markers are also associated with pre-eclampsia [50], and with a recent review of clinical factors associated with the condition [51].

Currently, although there are multiple models for the early prediction of pre-eclampsia, most use a wide range of maternal factors and biochemical markers; consequently, they are often complex and not easily translated into clinical practice [52]. At present, the best model is derived from an algorithm combining PlGF, measured at 10-13 weeks' gestation, with maternal MAP, uterine Doppler findings and an extensive suite of maternal data (age, weight, height, racial origin, interpregnancy interval, gestational age at delivery and presence/absence of pre-eclampsia in previous pregnancies, method of conception and presence/absence of chronic hypertension, diabetes mellitus, systemic lupus erythematosus and antiphospholipid syndrome) [53, 54]. Using this model, the predictive power of the maternal factors alone (as defined by Wright et al [53]) is significantly improved by addition of PIGF, MAP and uterine Doppler data; likewise, that of PIGF alone is improved by the other variables [54]. A simpler model to predict pre-eclampsia, as described in the current study (using three simple maternal characteristics and a single plasma biomarker), may be more convenient and widely applicable.

The strengths of our study include its longitudinal design, well-defined time points, initial collection of samples early in pregnancy (long before the onset of pre-eclampsia) and inclusion of a control group of non-diabetic women without preeclampsia for reference. New biomarkers for pre-eclampsia that operate early in pregnancy are proposed, and are urgently needed: they may elucidate pathogenic mechanisms for preeclampsia and its long-term sequelae. The main limitation is the small sample size in relation to the number of candidate biomarkers. It is both a strength and weakness that all participants were free of hypertension and microalbuminuria at the first trimester: this avoids the interference of prior complications in the search for biomarkers of pre-eclampsia, but has the disadvantage that the women may not be truly representative of the overall type 1 diabetic population. For the future, the data must be confirmed in a larger cohort. Future research should also determine whether the adipokine biomarkers might be useful in predicting pre-eclampsia even before a woman with type 1 diabetes becomes pregnant, since it is likely that first-trimester data reflect inherent maternal characteristics that are present before conception.

In conclusion, we suggest that circulating adipokines, leptin in particular, are associated with future pre-eclampsia as early as the first trimester in women with type 1 diabetes. The data are consistent with the hypothesis that, in women with type 1 diabetes, relatively high maternal insulin resistance in the first trimester (which could also be present prior to conception) is associated with the subsequent development of pre-eclampsia.

Acknowledgements The skilled and dedicated assistance of the following individuals for the clinical components of the study is acknowledged: M.K. Menard (University of North Carolina, Chapel Hill, NC, USA); J. Cole (Spartanburg Regional Hospital, Spartanburg, SC, USA); C. Knight, J. Conn, S. Hiscock, J. Oats and P. Wein (University of Melbourne, VIC, Australia); A. Dashti and M. Leyva (University of Oklahoma, Oklahoma City, OK, USA); H. Scholz (University of Oslo, Oslo, Norway); and J. R. Stanley (Mercy Health Center, Oklahoma City, OK, USA). We thank D. McCance (Queen's University, Belfast, UK) for helpful discussions.

Data availability The dataset is not publicly available for reasons of patient confidentiality. Please contact the authors for further information.

Funding This work was supported by Research Grants from the Juvenile Diabetes Research Foundation (JDRF 1-2001-844) and Novo Nordisk to TJL, and by National Institutes of Health (National Center for Research Resources) Grants M01-RR-1070 and M01 RR-14467 to the General Clinical Research Centers at the Medical University of South Carolina and University of Oklahoma Health Sciences Center, respectively. Support from Novo Nordisk enabled the participation of the Barbara Davis Diabetes Center for Childhood Diabetes at the University of Colorado, Denver, CO, USA.

Duality of interest The authors declare that there is no duality of interest associated with this manuscript.

Contribution statement All authors made significant contributions to the study concept, design and acquisition of data. CBK, CEA, CCP and TJL undertook the statistical analysis of the data, and all authors engaged in its interpretation. CBK and TJL drafted the manuscript and all authors participated critically in its revision. All authors approved the final version to be published, and agree to be accountable for all aspects of the work. 


\section{References}

1. Brown MA, Lindheimer MD, de Swiet M, Van Assche A, Moutquin JM (2001) The classification and diagnosis of the hypertensive disorders of pregnancy: statement from the International Society for the Study of Hypertension in Pregnancy (ISSHP). Hypertens Pregnancy 20:IX-XIV

2. Roberts JM, Gammill HS (2005) Preeclampsia: recent insights. Hypertension 46:1243-1249

3. American College of Obstetricians and Gynecologists; Task Force on Hypertension in Pregnancy (2013) Report. Obstet Gynecol 122: $1122-1131$

4. Persson M, Norman M, Hanson U (2009) Obstetric and perinatal outcomes in type 1 diabetic pregnancies: a large, population-based study. Diabetes Care 32:2005-2009

5. Briana DD, Malamitsi-Puchner A (2009) Reviews: adipocytokines in normal and complicated pregnancies. Reprod Sci 16:921-937

6. Schindler K, Vila G, Hoppichler F et al (2012) The impact of type 2 diabetes on circulating adipokines in patients with metabolic syndrome. Obes Facts 5:270-276

7. Syed Ikmal SI, Zaman Huri H, Vethakkan SR, Wan Ahmad WA (2013) Potential biomarkers of insulin resistance and atherosclerosis in type 2 diabetes mellitus patients with coronary artery disease. Int J Endocrinol 2013:698567

8. DeFronzo RA, Hendler R, Simonson D (1982) Insulin resistance is a prominent feature of insulin-dependent diabetes. Diabetes 31 : 795-801

9. Williams KV, Erbey JR, Becker D, Arslanian S, Orchard TJ (2000) Can clinical factors estimate insulin resistance in type 1 diabetes? Diabetes 49:626-632

10. Kilpatrick ES, Rigby AS, Atkin SL (2007) Insulin resistance, the metabolic syndrome, and complication risk in type 1 diabetes: "double diabetes" in the Diabetes Control and Complications Trial. Diabetes Care 30:707-712

11. Cleland SJ, Fisher BM, Colhoun HM, Sattar N, Petrie JR (2013) Insulin resistance in type 1 diabetes: what is 'double diabetes' and what are the risks? Diabetologia 56:1462-1470

12. Bjornstad P, Snell-Bergeon JK, Nadeau KJ, Maahs DM (2015) Insulin sensitivity and complications in type 1 diabetes: new insights. World J Diabetes 6:8-16

13. Kirwan JP, Hauguel-De Mouzon S, Lepercq J et al (2002) TNFalpha is a predictor of insulin resistance in human pregnancy. Diabetes 51:2207-2213

14. Hauth JC, Clifton RG, Roberts JM et al (2011) Maternal insulin resistance and preeclampsia. Am J Obstet Gynecol 204:327.e321327.e326

15. Wolf M, Sandler L, Munoz K, Hsu K, Ecker JL, Thadhani R (2002) First trimester insulin resistance and subsequent preeclampsia: a prospective study. J Clin Endocrinol Metab 87:1563-1568

16. Seely EW, Solomon CG (2003) Insulin resistance and its potential role in pregnancy-induced hypertension. J Clin Endocrinol Metab 88:2393-2398

17. Parretti E, Lapolla A, Dalfra M et al (2006) Preeclampsia in lean normotensive normotolerant pregnant women can be predicted by simple insulin sensitivity indexes. Hypertension 47:449-453

18. Persson M, Pasupathy D, Hanson U, Westgren M, Norman M (2012) Pre-pregnancy body mass index and the risk of adverse outcome in type 1 diabetic pregnancies: a population-based cohort study. BMJ Open 2:e000601

19. Kirwan JP, Huston-Presley L, Kalhan SC, Catalano PM (2001) Clinically useful estimates of insulin sensitivity during pregnancy. Validation studies in women with normal glucose tolerance and gestational diabetes mellitus. Diabetes Care 24: $1602-1607$
20. Ryan EA, O'Sullivan MJ, Skyler JS (1985) Insulin action during pregnancy. Studies with the euglycemic clamp technique. Diabetes 34:380-389

21. Xiang AH, Peters RK, Trigo E, Kjos SL, Lee WP, Buchanan TA (1999) Multiple metabolic defects during late pregnancy in women at high risk for type 2 diabetes. Diabetes 48:848-854

22. Masuyama $\mathrm{H}$, Inoue $\mathrm{S}$, Hiramatsu $\mathrm{Y}$ (2011) Retinol-binding protein 4 and insulin resistance in preeclampsia. Endocr J 58:47-53

23. Deng Y, Scherer PE (2010) Adipokines as novel biomarkers and regulators of the metabolic syndrome. Ann N Y Acad Sci 1212:E1-E19

24. Wauters M, Considine RV, Van Gaal LF (2000) Human leptin: from an adipocyte hormone to an endocrine mediator. Eur J Endocrinol 143:293-311

25. Hauguel-de Mouzon S, Lepercq J, Catalano P (2006) The known and unknown of leptin in pregnancy. Am J Obstet Gynecol 194: $1537-1545$

26. Randeva HS, Vatish M, Tan BK et al (2006) Raised plasma adiponectin levels in type 1 diabetic pregnancies. Clin Endocrinol 65:17-21

27. Nien JK, Mazaki-Tovi S, Romero R et al (2007) Adiponectin in severe preeclampsia. J Perinat Med 35:503-512

28. Fasshauer M, Waldeyer T, Seeger J et al (2008) Circulating highmolecular-weight adiponectin is upregulated in preeclampsia and is related to insulin sensitivity and renal function. Eur J Endocrinol 158:197-201

29. Miehle K, Stepan H, Fasshauer M (2012) Leptin, adiponectin and other adipokines in gestational diabetes mellitus and pre-eclampsia. Clin Endocrinol 76:2-11

30. Scifres CM, Catov JM, Simhan H (2012) Maternal serum fatty acid binding protein 4 (FABP4) and the development of preeclampsia. J Clin Endocrinol Metab 97:E349-E356

31. Fuseya T, Furuhashi M, Yuda S et al (2014) Elevation of circulating fatty acid-binding protein 4 is independently associated with left ventricular diastolic dysfunction in a general population. Cardiovasc Diabetol 13:126

32. Scifres CM, Chen B, Nelson DM, Sadovsky Y (2011) Fatty acid binding protein 4 regulates intracellular lipid accumulation in human trophoblasts. J Clin Endocrinol Metab 96:E1083-E1091

33. Steppan CM, Bailey ST, Bhat S et al (2001) The hormone resistin links obesity to diabetes. Nature 409:307-312

34. Yang Q, Graham TE, Mody N et al (2005) Serum retinol binding protein 4 contributes to insulin resistance in obesity and type 2 diabetes. Nature 436:356-362

35. Graham TE, Yang Q, Bluher M et al (2006) Retinol-binding protein 4 and insulin resistance in lean, obese, and diabetic subjects. N Engl J Med 354:2552-2563

36. Yu Y, Jenkins AJ, Nankervis AJ et al (2009) Anti-angiogenic factors and pre-eclampsia in type 1 diabetic women. Diabetologia 52: $160-168$

37. Basu A, Yu JY, Jenkins AJ et al (2015) Trace elements as predictors of preeclampsia in type 1 diabetic pregnancy. Nutr Res 35:421-430

38. DeLong ER, DeLong DM, Clarke-Pearson DL (1988) Comparing the areas under two or more correlated receiver operating characteristic curves: a nonparametric approach. Biometrics 44:837-845

39. Pencina MJ, D'Agostino RB Sr, D'Agostino RB Jr, Vasan RS (2008) Evaluating the added predictive ability of a new marker: from area under the ROC curve to reclassification and beyond. Stat Med 27:157-172, discussion 207-212

40. Pencina MJ, D'Agostino RB Sr, Steyerberg EW (2011) Extensions of net reclassification improvement calculations to measure usefulness of new biomarkers. Stat Med 30:11-21

41. Jin J, Peng DQ, Yuan SG et al (2010) Serum adipocyte fatty acid binding proteins and adiponectin in patients with coronary artery disease: the significance of A-FABP/adiponectin ratio. Clin Chim Acta 411:1761-1765 
42. Lekva T, Michelsen AE, Aukrust P, Henriksen T, Bollerslev J, Ueland T (2017) Leptin and adiponectin as predictors of cardiovascular risk after gestational diabetes mellitus. Cardiovasc Diabetol 16:5

43. Molvarec A, Szarka A, Walentin S et al (2011) Serum leptin levels in relation to circulating cytokines, chemokines, adhesion molecules and angiogenic factors in normal pregnancy and preeclampsia. Reprod Biol Endocrinol 9:124

44. Sagawa N, Yura S, Itoh H et al (2002) Role of leptin in pregnancya review. Placenta 23 Suppl A:S80-S86

45. Wotherspoon AC, Young IS, McCance DR et al (2016) Serum fatty acid binding protein 4 (FABP4) predicts pre-eclampsia in women with type 1 diabetes. Diabetes Care 39:1827-1829

46. Fasshauer M, Seeger J, Waldeyer T et al (2008) Serum levels of the adipokine adipocyte fatty acid-binding protein are increased in preeclampsia. Am J Hypertens 21:582-586

47. Shangguan X, Liu F, Wang H, He J, Dong M (2009) Alterations in serum adipocyte fatty acid binding protein and retinol binding protein- 4 in normal pregnancy and preeclampsia. Clin Chim Acta 407:58-61

48. Cortelazzi D, Corbetta S, Ronzoni S et al (2007) Maternal and foetal resistin and adiponectin concentrations in normal and complicated pregnancies. Clin Endocrinol 66:447-453
49. Nanda S, Nikoletakis G, Markova D, Poon LC, Nicolaides KH (2013) Maternal serum retinol-binding protein-4 at 11-13 weeks' gestation in normal and pathological pregnancies. Metabolism 62: 814-819

50. Ringholm L, Damm JA, Vestgaard M, Damm P, Mathiesen ER (2016) Diabetic nephropathy in women with preexisting diabetes: from pregnancy planning to breastfeeding. Curr Diab Rep 16(2):12

51. Vestgaard M, Sommer MC, Ringholm L, Damm P, Mathiesen ER (2017) Prediction of preeclampsia in type 1 diabetes in early pregnancy by clinical predictors: a systematic review. J Matern Fetal Neonatal Med DOI: 10.1080/14767058.2017.1331429

52. Kenny LC, Black MA, Poston L et al (2014) Early pregnancy prediction of preeclampsia in nulliparous women, combining clinical risk and biomarkers: the Screening for Pregnancy Endpoints (SCOPE) international cohort study. Hypertension 64:644-652

53. Wright D, Syngelaki A, Akolekar R, Poon LC, Nicolaides KH (2015) Competing risks model in screening for preeclampsia by maternal characteristics and medical history. Am J Obstet Gynecol 213:62.e61-e10

54. O'Gorman N, Wright D, Syngelaki A et al (2016) Competing risks model in screening for preeclampsia by maternal factors and biomarkers at 11-13 weeks gestation. Am J Obstet Gynecol 214: 103.e101-103.e112 